\title{
A Viral Infection Model with Lytic Immune Response and Periodic Medication Strategy
}

\author{
Baodan Tian ${ }^{1,2}$, Shouming Zhong ${ }^{1}$, Liu Yang ${ }^{1}$, Xianqing Liu ${ }^{1}$ \\ ${ }^{1}$ School of mathematical sciences, University of Electronic Science and Technology of China, Chengdu, 61173, China \\ ${ }^{2}$ School of science, School of science, Southwest University of Science and Technology, Mianyang, 621010, China \\ tommycdut@163t.com, tianbaodan@swust.edu.cn
}

\begin{abstract}
In this paper, a viral infection SVI model with lytic immune response is studied. What's more, in order to control the quantity of the virus population, periodic medication strategy is introduced to the classical SVI model. By some mathematical analysis techniques, an important threshold $\mathfrak{R}_{0}$ is found for the system. It is proved that the virus-free periodic solution (VFPS for short) is globally attractive if $\Re_{0}<1$, while the virus will be permanent if $\mathfrak{R}_{0}>1$. Finally, numerical simulation examples and discussions are presented to support our theoretical results.

Index Terms - VFPS, threshold, globally attractive, permanent .
\end{abstract}

\section{Introduction}

It is known that many new epidemics such as AIDS, SARS, HIV, HBV, HCV and H1N1, etc. appear and spread between humans and animals. Therefore, from mathematical and ecological point of view, the study on the effect of epidemic disease is a very important and interesting subject. And mathematical modelling is valuable and efficient in understanding the dynamics of the ecological system, epidemic transmission, viral infection and so on. Moreover, mathematical models can provide insights into the dynamics of viral load in vivo and is very helpful for clinical treatment. Especially, the models of combination therapy provide very important meaning for the cure of HIV, HBV and HCV. However, infection by HIV-1 and HCV has many puzzling quantitative features. For example, there is an average 10 years between infection with the virus and the AIDS in adults. The reason for this time lag remains largely unknown, although it seems tied to changes in the number of circulating $\mathrm{CD} 4+\mathrm{T}$ cells. The major target of HIV infection is a class of lymphocytes, or white blood cells, known as CD4+ T cells. These cells secrete growth and differentiation factors that are required by other cell populations in the immune system, and hence these cells are called "helper T cells" (see [1]).

In order to investigate the role of direct lytic and nonlytic inhibition of viral replication by immune cells in viral infections, Bartholdy et al. [2] and Wodarz et al. [3] constructed a mathematical model describing the basic dynamics of the interaction between susceptible host cells, a virus population, and immune response, which is described by the following differential equations (1),

$$
\left\{\begin{array}{l}
x^{\prime}(t)=s-d x(t)-\frac{\beta x(t) y(t)}{1+q z(t)} \\
y^{\prime}(t)=\frac{\beta x(t) y(t)}{1+q z(t)}-\alpha y(t)-p y(t) z(t) \\
z^{\prime}(t)=c y(t) z(t)-b z(t)
\end{array}\right.
$$

Recently, Song et. al studied a delayed viral infection model with lytic immune response in [1]. They studied the effect of time delay on the stability of the equilibrium of the system, and get sufficient conditions for stability switch of positive equilibrium.

Enlightened by above literatures, in the present paper, we will introduce periodic medication strategy to the classical model (1) (we call it SVI model for short in the following). The main purpose of the paper is finding a efficient medication strategy so that we could control the virus population by changing the period of the medication or strengthening the medication, and the model is as follows,

$$
\left\{\begin{array}{l}
S^{\prime}(t)=r-d S(t)-\frac{\beta S(t) V(t)}{1+q I(t)} \\
V^{\prime}(t)=\frac{\beta S(t) V(t)}{1+q I(t)}-\alpha V(t)-p V(t) I(t) \\
I^{\prime}(t)=c V(t) I(t)-b I(t) \\
S\left(t^{+}\right)=\left(1-\delta_{1}\right) S(t) \\
V\left(t^{+}\right)=\left(1-\delta_{2}\right) V(t) \\
I\left(t^{+}\right)=\left(1-\delta_{3}\right) I(t)
\end{array}\right\} t=n T, n=1,2, \cdots
$$

with initial condition

$$
S\left(0^{+}\right)=S_{0}, V\left(0^{+}\right)=V_{0}, I\left(0^{+}\right)=I_{0}
$$

where $S(t)$ is the number of susceptible host cells, $V(t)$ is the number of virus population and $I(t)$ is the number of immune responses; susceptible host cells are generated at a rate $r$, die at rate of $d S(t)$ and become infected by virus at rate of $\beta S(t) V(t)$ without the immune response; to model nonlytic antiviral, viral replication is inhibited by the immune response at a rate $1+q I(t)$; infected cells die at a rate ay and killed by the immune system at a rate $p V(t) I(t)$ for modeling lytic effecter mechanisms; the immune response is assumed to get stronger at a rate proportional to the number of infected cells, $c V(t) I(t)$; also decay exponentially at a rate proportional to its current strength, $b I(t)$; the parameter $p(>c)$ expresses the 
strength of the lytic component, whereas the parameter $q$ expresses the efficacy of the nonlytic component.

\section{Preliminaries}

Let $R_{+}=[0, \infty), R_{+}^{3}=\left\{(x, y, z) \in R^{3} \mid x, y, z \geq 0\right\}, \Omega=\operatorname{int} R_{+}^{3}$. Denote as $f=\left(f_{1}, f_{2}, f_{3}\right)$ the map defined by the right hand sides of the first, second and third equation of the system(2).

Let $V: R_{+} \times R_{+}^{3} \rightarrow R_{+}$is said to belong to class $V_{0}$ if

(1) $V$ is continuous in $(n T,(n+1) T] \times R_{+}^{3}$, and for each $x \in R^{3}, n \in N, \lim _{(t, y) \rightarrow\left(n T^{+}, x\right)} V(t, y)=V\left(n T^{+}, x\right)$ exists;

(2) $V$ is locally Lipschitzian for $x$.

Lemma 1. Comparison theorem of impulsive differential equations, we omit it here and more details can be seen in[4]. Lemma 2(see [5]). Consider the following subsystem of (2)

$$
\left\{\begin{array}{l}
S^{\prime}(t)=r-d S(t), \quad t \neq n T \\
S\left(t^{+}\right)=\left(1-\delta_{1}\right) S(t), \quad t=n T, n=1,2, \cdots
\end{array}\right.
$$

There exists a unique periodic solution of system (5)

$S_{0}(t)=\frac{r}{d}\left(1-\frac{\delta_{1} \exp (-d(t-n T))}{1-\left(1-\delta_{1}\right) \exp (-d T)}\right)$

which is globally asymptotically stable.

Lemma 3. For each solution $(S(t), V(t), I(t))$ of system (2), there exists a constant $M>0$ such that $S(t) \leq M, V(t) \leq M, I(t) \leq M$ for with $\mathrm{t}$ large enough.

Proof. Let $N(t)=S(t)+V(t)+I(t)$,

$$
\text { then } N^{\prime}(t) \leq r-d S(t)-\alpha V(t)-b I(t) \leq r-D N(t)
$$

where $D=\min \{\alpha, b, d\}, N\left(t^{+}\right)=\left(1-\delta_{1}\right) S(t)+\left(1-\delta_{2}\right) V(t)+\left(1-\delta_{3}\right) I(t) \leq N(t)$ which leads to

$$
\begin{aligned}
& \left\{\begin{array}{l}
N^{\prime}(t)+D N(t) \leq r, \quad t \neq n T \\
N\left(t^{+}\right) \leq N(t), \quad t=n T
\end{array}\right. \\
& \therefore N(t) \leq N\left(0^{*}\right) e^{-D t}+\frac{r}{D}\left(1-e^{-D t}\right) \rightarrow \frac{r}{D} \triangleq M
\end{aligned}
$$

That is to say, there exists a $M>0$ such that $S(t) \leq M, V(t) \leq M, I(t) \leq M$ with $t$ large enough.

\section{Global behavior of VFPS}

At first of this section, we denote

$$
\Re_{0} \triangleq \frac{d\left(e^{d T}-\left(1-\delta_{1}\right)\right.}{r \beta \delta_{1}\left(e^{d T}-1\right)}\left(d \ln \left(1-\delta_{2}\right)+T(\beta r-\alpha d)\right),
$$

then we have following theorem for the global attractivity of the virus-free periodic solution firstly. Theorem 1 . If $\mathfrak{R}_{0}<1$, then the virus-free periodic solution (VFPS) $\left(S_{0}(t), 0,0\right)$ of system (2) is globally attractive. Proof. Since $\mathfrak{R}_{0}<1$ holds, then we can choose $\varepsilon>0$ small enough such that

$$
\eta_{1}=\left(1-\delta_{2}\right) \exp \left(\int_{0}^{T}\left(\beta\left(S_{0}(t)+\varepsilon\right)-\alpha\right) d t\right)<1
$$

From the first and the fourth equation of system (2),

$$
\left\{\begin{array}{l}
S^{\prime}(t) \leq r-d S(t), \quad t \neq n T \\
S\left(t^{+}\right)=\left(1-\delta_{1}\right) S(t), \quad t=n T, n=1,2, \cdots
\end{array}\right.
$$

By lemma 1 and lemma 2, there exists a $T_{1}>0$ such that

$$
s(t) \leq S_{0}(t)+\varepsilon, \text { when } t>T_{1}
$$

On the other hand, when $t>T_{1}$ from the second equation of system (2) we have $V^{\prime}(t) \leq\left(\beta\left(S_{0}(t)+\varepsilon\right)-\alpha\right) V(t)$ which yields

$$
\frac{V^{\prime}(t)}{V(t)} \leq \beta\left(S_{0}(t)+\varepsilon\right)-\alpha
$$

Integrate the inequality (10) on the interval $(n T,(n+1) T]$, we have that

$$
\begin{aligned}
V((n+1) T) & \leq V\left(n T^{+}\right) \exp \left(\int_{n T}^{(n+1) T}\left(\beta\left(S_{0}(t)+\varepsilon\right)-\alpha\right) d t\right) \\
& =V(n T)\left(1-\delta_{2}\right) \exp \left(\int_{0}^{T}\left(\beta\left(S_{0}(t)+\varepsilon\right)-\alpha\right) d t\right) \\
& =V(n T) \eta_{1}
\end{aligned}
$$

which leads to $V(n T) \leq V\left(0^{+}\right) \eta_{1}^{n} \rightarrow 0$, as $n \rightarrow \infty$ Since $0 \leq V(t) \leq V(n T), \quad t \in((n-1) T, n T]$, then

$$
\lim _{t \rightarrow \infty} V(t)=0
$$

Thus, for any $0<\varepsilon_{1}<b / c$ small enough, there exists a $T_{2}>T_{1}$ such that $V(t)<\varepsilon_{1}$ when $t>T_{2}$, and by the third equation of system (2) we have

$$
I^{\prime}(t) \leq\left(c \varepsilon_{1}-b\right) I(t) \Rightarrow \frac{I^{\prime}(t)}{I(t)} \leq c \varepsilon_{1}-b
$$

Integrate (9) on the interval $(n T, t], t \in(n T,(n+1) T]$, we have

$$
\begin{aligned}
I(t) & \leq I\left(n T^{+}\right) \exp \left(\int_{n T}^{(n+1) T}\left(c \varepsilon_{1}-b\right) d t\right) \\
& \leq V_{0}\left(1-\delta_{2}\right)^{n} \exp \left(\left(c \varepsilon_{1}-b\right) T\right) \\
\therefore I(t) & \rightarrow 0, \text { as } n \rightarrow \infty \text {, i.e, } \lim _{t \rightarrow \infty} I(t)=0 .
\end{aligned}
$$

On the other hand, by the first and fourth equation of system (2) once again, we have that

$$
\left\{\begin{array}{l}
S^{\prime}(t) \geq r-\left(d+\beta \varepsilon_{1}\right) S(t), \quad t \neq n T \\
S\left(t^{+}\right)=\left(1-\delta_{1}\right) S(t), t=n T, n=1,2, \cdots
\end{array}\right.
$$

Also, by the comparison theorem and Lemma 2, there exists a $T_{3}>T_{2}$ such that

$$
S(t) \geq S_{0}^{\varepsilon_{1}}(t)-\varepsilon, \text { when } t>T_{3}
$$

where $S_{0}^{\varepsilon_{1}}(t)=\frac{r}{d+\beta \varepsilon_{1}}\left(1-\frac{\delta_{1} \exp \left(-\left(d+\beta \varepsilon_{1}\right)(t-n T)\right)}{1-\left(1-\delta_{1}\right) \exp \left(-\left(d+\beta \varepsilon_{1}\right) T\right)}\right)$ 
Combine inequality (9) and (15), for any $\varepsilon, \varepsilon_{1}>0$ small enough, there exists a $T_{3}>T_{2}$ such that $S_{0}^{z_{1}}(t)-\varepsilon \leq S(t) \leq S_{0}(t)+\varepsilon$ when $\mathrm{t}>T_{3}$, Let $\varepsilon_{1} \rightarrow 0$, we have

$$
S_{0}(t)-\varepsilon \leq S(t) \leq S_{0}(t)+\varepsilon, \text { when } t>T_{3}
$$

which implies

$$
\lim _{t \rightarrow \infty} S(t)=S_{0}(t)
$$

By (11), (13) and (17), we know that the $\operatorname{VFPS}\left(S_{0}(t), 0,0\right)$ of system (2) is globally attractive.

\section{Permanence}

In this section, we say the virus spread if the virus population persists above a certain positive level for a long period, and at first we give the definition of the permanence of the virus population.

Definition. The virus population $V(t)$ is said to be permanent if there are constant $V_{M}>V_{L}>0$ such that $V_{L} \leq \liminf _{t \rightarrow \infty} V(t) \leq \limsup _{t \rightarrow \infty} V(t) \leq V_{M}$ for any solution $(S(t), V(t), I(t))$ of system (2).Theorem 2 . If $\mathfrak{R}_{0}>1$, then virus population is permanent.

Proof. At first, for $l \in(0, b / c)$ is small enough, we claim

$$
\limsup _{t \rightarrow \infty} V(t) \geq l
$$

Suppose that (15) is not true, then there exists a $T_{4} \geq 0$ such that $V(t)<l$ for all $t \geq T_{4}$, and from the first and the fourth equation of system (2) we have

$$
\left\{\begin{array}{l}
S^{\prime}(t) \geq r-(d+\beta l) S(t), \quad t \neq n T \\
S\left(t^{+}\right)=\left(1-\delta_{1}\right) S(t), t=n T, n=1,2, \cdots
\end{array}\right.
$$

Then by comparison theorem and Lemma 2, there exists a $T_{5}>T_{4}$ such that

$$
S(t) \geq S_{0}^{l}(t)-\varepsilon, \text { when } t>T_{5}
$$

where

$$
S_{0}^{l}(t)=\frac{r}{d+\beta l}\left(1-\frac{\delta_{1} \exp (-(d+\beta l)(t-n T))}{1-\left(1-\delta_{1}\right) \exp (-(d+\beta l) T)}\right)
$$

And it is easy to see

$$
S_{0}^{l}(t)>\frac{r}{d+\beta l}\left(1-\frac{\delta_{1}}{1-\left(1-\delta_{1}\right) \exp (-(d+\beta l) T)}\right) \triangleq S_{L}
$$

Also, by the third and the last equation of system (2),

$$
\left\{\begin{array}{l}
I^{\prime}(t) \leq\left(c \varepsilon_{1}-b\right) I(t), \quad t \neq n T \\
I\left(t^{+}\right)=\left(1-\delta_{3}\right) I(t), t=n T, n=1,2, \cdots
\end{array}\right.
$$

Since $l \in(0, b / c)$ is small enough, then similar as the proof of (13) in Theorem 1 , and there exists a $T_{6}>T_{5}$, such that

$$
I(t)<\varepsilon \text { when } t>T_{6}
$$

Then by the second equation of system (2),

$$
V^{\prime}(t)>\left(\frac{\beta\left(S_{0}^{l}(t)-\varepsilon\right)}{1+q \varepsilon}-\alpha-p \varepsilon\right) V(t)
$$

Because of $\mathfrak{R}_{0}>1$ and $l \in(0, b / c)$ is small enough, we can choose $\varepsilon>0$ small enough such that

$\eta_{2}=\left(1-\delta_{2}\right) \exp \left(\int_{0}^{T}\left(\frac{\beta\left(S_{0}^{l}(t)-\varepsilon\right)}{1+q \varepsilon}-\alpha-p \varepsilon\right) d t\right)>1$, and $\sigma=\frac{\beta\left(S_{L}-\varepsilon\right)}{1+q \varepsilon}-\alpha-p \varepsilon>0$.

Integrate (23) on the interval $(n T,(n+1) T]$, we have

$V((n+1) T)>V(n T)\left(1-\delta_{2}\right) \exp \left(\int_{0}^{T}\left(\frac{\beta\left(S_{0}^{l}(t)-\varepsilon\right)}{1+q \varepsilon}-\alpha-p \varepsilon\right) d t\right)=V(n T) \eta_{2}$

which yields $V(n T)>V\left(0^{+}\right) \eta_{2}^{n} \rightarrow \infty$, as $n \rightarrow \infty$

which is a contradiction with the boundedness of $V(t)$.

Therefore, there exists a $t_{1}>0$ such that

$$
V\left(t_{1}\right)>l \text {, i.e, } \limsup _{x \rightarrow \infty} V(t)>l .
$$

Secondly, if the inequality $V(t)>l$ holds for all $t>t_{1}$, then our aim is obtained. So we only need to consider the case that $V(t)$ leaves from the compact region $R=\{(S(t), V(t), I(t)) \mid V(t) \leq l\}$ and reenter it again.

If $t^{*}=n_{1} T, n_{1} \in N$, according to (24) there must exist a $n_{1} \in N$ such that $V(t) \geq l, t \in\left[t_{1}, t^{*}\right]$ and $\left(1-\delta_{2}\right) l \leq V\left(t^{*+}\right)=\left(1-\delta_{2}\right) V\left(t^{*}\right)<l$

Then we can choose $n_{2}, n_{3} \in N$ and $\varepsilon>0$ small enough such that $\left(1-\delta_{2}\right)^{n_{2}} \exp \left(n_{2} \sigma T\right)>1$.

Let $\bar{T}=\left(n_{2}+n_{3}\right) T$, then we claim that there exists a $t_{2} \in\left(t^{*}, t^{*}+\bar{T}\right)$, such that $V\left(t_{2}\right) \geq l$ holds.

Otherwise, if $V(t)<l$ holds for all $t \in\left(t^{*}, t^{*}+\bar{T}\right)$, then (19)-(23) hold, and by (20) we have

$$
\frac{V^{\prime}(t)}{V(t)}>\frac{\beta\left(S_{L}-\varepsilon\right)}{1+q \varepsilon}-\alpha-p \varepsilon
$$

Integrate (25) on the interval $\left(t^{*},\left(n_{1}+1\right) T\right] \cup\left(\left(n_{1}+1\right) T\right.$, $\left.\left(n_{1}+2\right) T\right] \bigcup \cdots \cup\left(\left(n_{1}+n_{2}-1\right) T, t^{*}+n_{2} T\right]$, we have

$$
\begin{aligned}
V\left(t^{*}+n_{2} T\right) & >V\left(t^{*+}\right) \exp \left(\int_{t^{*}}^{t^{*}+n_{2} T}\left(\frac{\beta\left(S_{0}^{l}(t)-\varepsilon\right)}{1+q \varepsilon}-\alpha-p \varepsilon\right) d t\right) \\
& \geq l\left(1-\delta_{2}\right)^{n} \exp \left(n_{2} \sigma T\right)>l
\end{aligned}
$$

which is a contradiction.

Thus, if we denote $\bar{t}=\inf _{t \geq t^{*}}\{V(t)>l\}$, then $V(t)<l$ for all $t \in\left(t^{*}, \bar{t}\right)$ and $V(\bar{t})=l$.

In the same way by (25) when $t \in\left(\bar{t}, t^{*}+\bar{T}\right]$,

$$
\begin{aligned}
& V(t)>V(\bar{t}) \exp \left(\int_{\bar{t}}^{t}\left(\frac{\beta\left(S_{0}^{l}(t)-\varepsilon\right)}{1+q \varepsilon}-\alpha-p \varepsilon\right) d t\right) \\
& \therefore V(t) \geq l\left(1-\delta_{2}\right)^{\kappa_{2}+\pi_{3}} \exp \left(\left(n_{2}+n_{3}\right) \sigma T\right) \triangleq V_{L}
\end{aligned}
$$


Since $V\left(t^{*}+\bar{T}\right) \geq V_{L}$, repeat above process we have $V(t) \geq V_{L}$ for all $t>\bar{t}$.

On the other hand, if $t^{*} \neq n_{1} T, n_{1} \in N$, it is very similar to prove that there exists a $\tilde{t}>0$ such that $V(t) \geq V_{L}$ for all $t>\bar{t}$.

And as the page is limited, we omit this process.

That is to say, $\liminf _{t \rightarrow \infty} V(t) \geq V_{L}$ holds, combined with Lemma 3, the virus population is permanent.

\section{Numeric Simulations and discussions}

In this paper, we have analyzed the global attractivity of the virus-free periodic solution of a viral infection SVI model with lytic immune response and periodic medication strategy. And we obtained an important critical value $\mathfrak{R}_{0}$ successfully, it is shown that the VFPS is globally attractive and the virus will be eradicated finally when $\mathfrak{R}_{0}<1$ in section 3 , while it is shown that the virus will be permanent if $\mathfrak{R}_{0}>1$ in section 4 .

Thus, in order to verify above theoretical results, we will give some numerical simulations in the following.

For system (2) if we choose

$r=0.8, d=0.1, \alpha=0.3, \beta=0.3, p=0.8, q=1.0, b=0.1$,

$c=0.6, T=1, \delta_{1}=0.2, \delta_{2}=0.4, \delta_{3}=0.1$

with initial conditions

$$
S_{0}=0.5, V_{0}=0.3, I_{0}=0.2
$$

and by a direct computation we have

$\mathfrak{R}_{0}=\frac{d\left(e^{d T}-\left(1-\delta_{1}\right)\right.}{r \beta \delta_{1}\left(e^{d T}-1\right)}\left(d \ln \left(1-\delta_{2}\right)+T(\beta r-\alpha d)\right) \approx 0.9607<1$

One hand, by Theorem 1, the VFPS $\left(S_{0}(t), 0,0\right)$ of system (2) is globally attractive; On the other hand, by Maple software, we can plot the time series diagram for $(S(t), V(t), I(t))$ as Fig.1, and one can see that both the virus population and the immune response (CD4+T cells) will be eventually eradicated, but the susceptible host cells $S(t)$ can keep permanent.
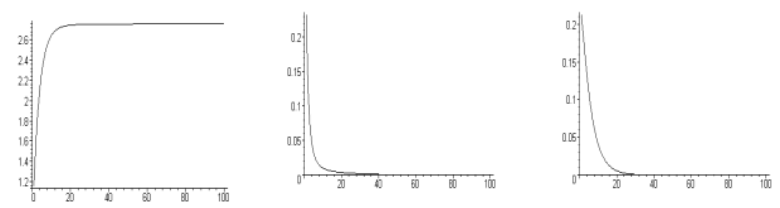

Fig. 1 Time series diagram for $(S(t), V(t), I(t))$ with $\mathrm{T}=1$.

If we only prolong the medication period, other parameters keep the same, such that $T=2$. By a simple computation we obtain

$\mathfrak{R}_{0}=\frac{d\left(e^{a t}-\left(1-\delta_{1}\right)\right.}{\left.r \beta \delta_{(} e^{a t}-1\right)}\left(d \ln \left(1-\delta_{2}\right)+T(\beta r-\alpha d)\right) \approx 1.4629>1$ One hand, by

Theorem 2, the virus population will be permanent; on the other hand, by Maple software, we can plot the time series diagram for $(S(t), V(t), I(t))$ as Fig.2, and one can see that both the virus population and the immune response (CD4+T cells) will be permanent.
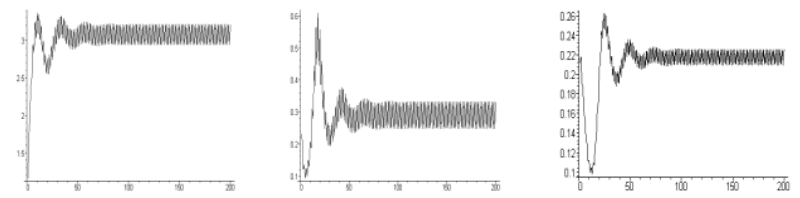

Fig. 2 Time series diagram for $(S(t), V(t), I(t))$ with $\mathrm{T}=2, \delta 2=0.4$.

Above two examples show that if we intend to eliminate the virus population effectively, we must choose suitable medication period, if the medication period is too long, the medication strategy might be failed. However, intuitively speaking, as long as we increase the intensity of each medication, i.e, increase the parameter $\delta_{i}(i=1,2,3)$, we might also be able to eradicate the viral population, but in fact is it right? And if we choose $\delta_{2}=0.7>0.4$ and other parameters keep the same, then

$\Re_{0}=\frac{d\left(e^{d T}-\left(1-\delta_{1}\right)\right.}{r \beta \delta_{1}\left(e^{d T}-1\right)}\left(d \ln \left(1-\delta_{2}\right)+T(\beta r-\alpha d)\right) \approx 1.1880>1$

Also, the virus population will be permanent by Theorem 1; and we can also plot the time series diagram as Fig.3 by Maple software. From Fig.3, we can see that the immune response (CD4+T cells) will be eventually eradicated while the virus population will still be permanent. And this phenomena tell us excessive medication strategy will not kill the virus effectively instead of eradicating the immune response.
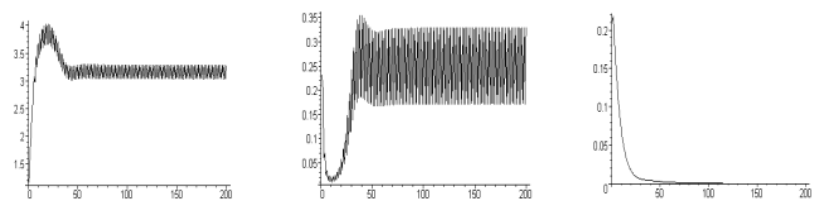

Fig.3 Time series diagram for $(S(t), V(t), I(t))$ with $\mathrm{T}=2, \delta 2=0.7$.

\section{References}

[1] Xinyu Song, Shaoli Wang and Jing Dong, "Stability properties and Hopf bifurcation of a delayed viral infection model with lytic immune response," Journal of Mathematical Analysis and Applications, vol.373, no.2, pp. 345-355, 2011.

[2] C. Bartholdy, J.P. Christensen, et. al, "Persistent virus infection despite chronic cytotoxic T-lymphocyte activation in Gamma interferon-deficient mice infection with lymphocytic choriomeningitis virus," Journal of Virology, vol.74, no.22, pp.10304-10311, 2000.

[3] D. Wodarz, J.P. Christensen, A.R. Thomsen, "The importance of lytic and nonlytic immune response in viral infections", Trends in Immunology, vol.23, no.4, pp. 194-200, 2002.

[4] V. Lakshmikanthamv, D. D. Simeonov, Theory of Impulsive Differential Equations. World Scientific, Singapore, 1989.

[5] Tailei Zhang, Zhidong Teng. "An SIRVS epidemic model with pulse vaccination strategy," Journal of Theoretical Biology, vol.250, no.2, pp.375-381, 2008. 